$18,07,14$

\title{
Моделирование электронной структуры композитного материала графен-поливинилиденфторид
}

\author{
(C) А.Г. Гасанов, А.А. Байрамов
}

Военная академия вооруженных сил, Баку, Азербайджан

E-mail: azad.bayramov@yahoo.com

(Поступила в Редакцию 24 апреля 2018 г.

В окончательной редакции 14 июня 2018 г.)

Построены теоретические модели ударопрочных композитных материалов на основе двухслойных графенов и многослойного поливинилиденфторида $\mathrm{C}_{124} \mathrm{H}_{40}+n\left(\mathrm{H}-\left(\mathrm{C}_{2} \mathrm{H}_{2} \mathrm{~F}_{2}\right)_{5}-\mathrm{H}\right)(n=\overline{1,8})$. Исследована электронная структура полуэмпирическим РМ3-методом, который является одним из вариантов метода молекулярных орбиталей. На основе теоретических моделей были вычислены орбитальные энергии, потенциалы ионизации, полные энергии электронов, прочность и т.д. данного материала. Рассмотрены перспективы использования этих материалов в военной области для изготовления сверхпрочных и легких бронежилетов.

Выполнение данной работы осуществлено за счет финансирования Военной академии вооруженных сил Азербайджанской Республики.

DOI: 10.21883/FTT.2019.01.46913.116

\section{1. Введение}

Графен представляют в виде одноатомного слоя атомов углерода, соединенных в двухмерной решетке $\sigma$ и $\pi$ связями, и находящегося в состоянии $s p^{2}$ гибридизации. Кристаллическая структура графена - гексагональная решетка, постоянная решетки - $0.246 \mathrm{~nm}[1,2,3]$. Атомы углерода в слое графена соединены между собой сильной ковалентной связью. В углероде три валентных электрона из четырех участвуют в создании $\sigma$-связи, а один валентный электрон - в создании $\pi$-связи. В состоянии $\pi$-связи перекрывание электронных облаков друг с другом меньше, чем в состоянии $\sigma$-связи. Поэтому, $\pi$-связи легко обрываются и $\pi$-электроны передвигаются вокруг всех атомных ядер внутри решетки. Это обеспечивает проводимость графена.

Графен обладает большой механической жесткостью $\sim 1 \mathrm{TPa}$ [3-7]. Помимо этого, звуковые волны в графене распространяются в три раза быстрее, чем в стали, а это значит, что графен может быстрее поглощать и рассеивать энергию акустических волн, которые могут создаваться под действием, например, пули, выпущенной из огнестрельного оружия. Графен эффективно замедляет пулю, предотвращая его дальнейшее проникновение. Поэтому, возникает реальная перспектива создания сверхпрочного и легкого пуленепробиваемого бронежилета на основе графеновых листов [8].

Листы графена рассеивают кинетическую энергию, вытягиваясь в форме конуса в месте соприкосновения с пулей, а затем трескаются и рассыпаются на осколки (см. рис. 1). Эти осколки также представляют угрозу для человека. Эту проблему можно решить применением многослойного графенового покрытия (это резко повысит его стоимость) или включением его в полимерную композитную структуру. В нашей работе мы рассмотрели композитную структуру на основе двухслойного графена и многослойной полимерной матрицы.

Двухслойный графен обладает большой механической прочностью $[5,6,7]$. Это свойство обеспечивает применение этих графеносодержащих материалов в широких областях, особенно в военной области. Поэтому, представляет большой интерес изучение двухслойных графено содержащих ударопрочных материалов. С целью защиты от осколков в качестве полимерной матрицы в композитном материале был рассмотрен полимерный материал поливинилиденфторид (ПВДФ или PVDF). С этой точки зрения, представляет большой интерес теоретического изучения композитного материала на основе двухслойного графена и полимерной матрицы $\mathrm{C}_{124} \mathrm{H}_{40}+n \mathrm{PVDF}$.

В настоящей работе приводятся результаты расчетов теоретических моделей композитного материала $\mathrm{C}_{124} \mathrm{H}_{40}+n\left(\mathrm{H}-\left(\mathrm{C}_{2} \mathrm{H}_{2} \mathrm{~F}_{2}\right)_{5}-\mathrm{H}\right) \quad(n=\overline{1,8})$ на основе двухслойного графена и многослойной полимер-

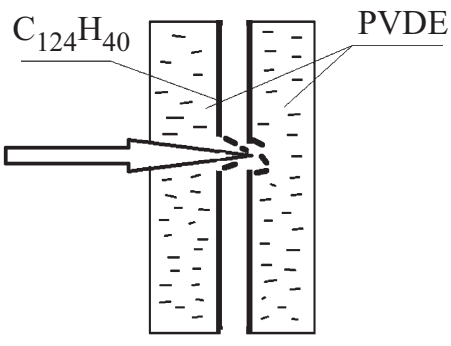

Рис. 1. Раздробление композитного материала на основе двухслойного графена в полимерной матрице $\mathrm{C}_{124} \mathrm{H}_{40}+2\left(\mathrm{H}-\mathrm{C}_{10}+\mathrm{H}_{10}+\mathrm{F}_{10}-\mathrm{H}\right)$ под действием удара пули. 
ной матрицы, и исследований электронной структуры данного материала, используя полуэмпирический метод РМ3 [9]. Были рассчитаны орбитальные энергии электронов $\varepsilon_{i}$. Для данного ударопрочного материала $\mathrm{C}_{124} \mathrm{H}_{40}+n\left(\mathrm{H}-\left(\mathrm{C}_{2} \mathrm{H}_{2} \mathrm{~F}_{2}\right)_{5}-\mathrm{H}\right)$, используя значения $\varepsilon_{i}$, были вычислены полные энергии электронов, значения потенциалов ионизации, исследованы некоторые механические, электрические и оптические характеристики. Для проведения расчетов использовались программы Mathcad, free evaluate version HyperChem Professional v7.5 и MS office Excel.

\section{2. Методика}

Математические моделирование различных характеристик ударопрочных материалов на основе графена и квантовомеханическое исследование имеет важное значение $[10,11,12]$. Такие теоретические расчеты проводят обычно методом молекулярных орбиталей (МО) [13-19].

Известно, что полуэмпирический метод РМ3 является простым вариантом метода молекулярных орбиталей (MO) [20]. В методе МО допускается, что в молекуле каждый электрон независимо движется в некотором эффективном поле, создаваемым ядрами молекул и другими электронами. Состояние электрона в молекуле описывается одноэлектронной волновой функцией, называемой молекулярным орбиталем [17,18,21,22]. Эти функции многоцентровые. Так, в их выражения входят расстояния электрона от различных атомных ядер. Существуют различные варианты нахождения молекулярных орбиталей. Один из них, метод нахождения молекулярного орбиталя (метод MO LCAO) $U_{i}$, как линейной комбинации атомных орбиталей атомов, входящих в молекулу

$$
U_{i}=\sum_{q=1}^{m} c_{q} \chi_{q} .
$$

Здесь: $c_{q i}-$ коэффициенты, $\chi_{q}$ - атомные орбитали, выбираемые как базисные функции. В работе в качестве атомных орбиталей были использованы функции Гаусca [23].

Коэффициенты находим решением системы уравнений

$$
\sum_{q}\left(H_{p q}-\varepsilon_{i} S_{p q}\right) c_{q i}=0 .
$$

Здесь:

$$
\begin{gathered}
H_{p q}=\int \chi_{p}^{\bullet} \hat{H}_{e f} \chi_{q} d V, \\
S_{p q}=\int \chi_{p}^{\bullet} \chi_{q} d V .
\end{gathered}
$$

$S_{p q}$ - интегралы перекрытия атомных орбиталей $\chi_{p}$ и $\chi_{q} . \hat{H}_{e f}$ оператор Гамильтона для электрона, движущегося в некотором эффективном поле молекулы, независимо от других электронов:

$$
\hat{H}_{e f}=-\frac{1}{2} \nabla^{2}+U(r) .
$$

Из-за того, что потенциальная функция $U(r)$ не известна в явном виде, невозможно точно вычислить $H_{p q}$. Они оцениваются с помощью определенных экспериментальных параметров. Диагональные элементы матрицы $H_{p q}$ берутся равными потенциалам ионизации соответствующих валентных состояний атомов с обратным знаком [23]

$$
\begin{aligned}
& (1 s|H| 1 s)=-0.499786 \text { a.u., } \\
& (2 s|C| 2 s)=-0.772096 \text { a.u., } \\
& (2 p|C| 2 p)=-0.419161 \text { a.u. }
\end{aligned}
$$

Не диагональные элементы $H_{p q}$ могут быть вычислены различными приближениями [7,11].

(2) представляет собой систему линейных однородных уравнений. Решая эту систему находим численные значения величин $\varepsilon_{i}$ и $c_{q i}$. Используя значения $\varepsilon_{i}$ можно вычислить значения полной энергии электрона и потенциала ионизации, а также исследовать свойства материала.

В предыдущих наших работах применяя полуэмпирический РМ3-метод были исследованы электронные структуры различных материалов [14-22]. Расчеты показывают, что в С-C-линейных цепочках и в графеноподобных кластерах, независимо от размера, равновесные расстояния $\mathrm{C}-\mathrm{C}$ оказываются равными $0.13 \mathrm{~nm}$ [11]. В данной работе расчитаны и построены теоретические модели двухслойного графена $\mathrm{C}_{124} \mathrm{H}_{40}$ (рис. 2) и композитного материала на основе двухслойного графена и многослойной полимерной (ПВДФ) матрицы $\mathrm{C}_{124} \mathrm{H}_{40}+\left(\mathrm{H}-\mathrm{C}_{10}+\mathrm{H}_{10}+\mathrm{F}_{10}-\mathrm{H}\right)$ (рис. 3). Были взяты: молекулярная масса двухслойного графена $\mathrm{C}_{124} \mathrm{H}_{40} \quad \mathrm{M}_{\mathrm{C}_{124} \mathrm{H}_{40}}=1529.6444 \mathrm{amu}$ [5], молекулярная масса ПВДФ $\mathrm{M}_{\mathrm{H}-\mathrm{C}_{10} \mathrm{H}_{10} \mathrm{~F}_{10}-\mathrm{H}}=322.186 \mathrm{amu}$. Расстояния $\mathrm{C}-\mathrm{C}$ были взяты $0.13 \mathrm{~nm}$ далее были проведены расчеты по методу РМ3. Расчеты проведены при следующих условиях: при неизменности молекулярной массы двухслойного графена $\mathrm{C}_{124} \mathrm{H}_{40}$, молекулярная масса ПВДФ

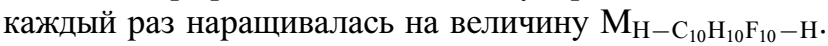

Для каждого объекта, электроны размещены по двое на энергетических уровнях, начиная с наиболее низкого уровня. Были определены энергии занятые электронами

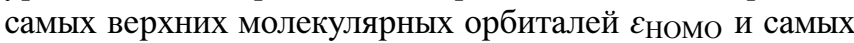
низких пустых молекулярных орбиталей $\varepsilon_{\text {LUMO }}$. Были

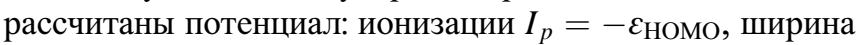
запрещенной зоны $E_{g}=\varepsilon_{\text {LUMO }}-\varepsilon_{\text {HOMO }}$ и прочность (твердый материал или мягкий) $\eta=0.5 E_{g}$. При $\eta<1 \mathrm{eV}$ материал считается мягким, при $\eta>1 \mathrm{eV}$ материал считается твердым.

Длина волны излучаемого фотона может быть рассчитана по формуле $\lambda=\left(\operatorname{ch} 10^{28}\right) /\left(1.6 E_{g}\right)(\mathrm{nm})$. Здесь $h-$ постоянная Планка, $c$ - скорость света в вакууме, $E_{g}-$ ширина запрещенной зона в $\mathrm{eV}$.

Стабильность материала была рассчитана по формуле

$$
\Delta E=E-\sum_{A} E_{A} .
$$

Здесь $E$ - полная энергия системы, $E_{A}-$ полная энергия свободного атома А, входящего в систему, и 

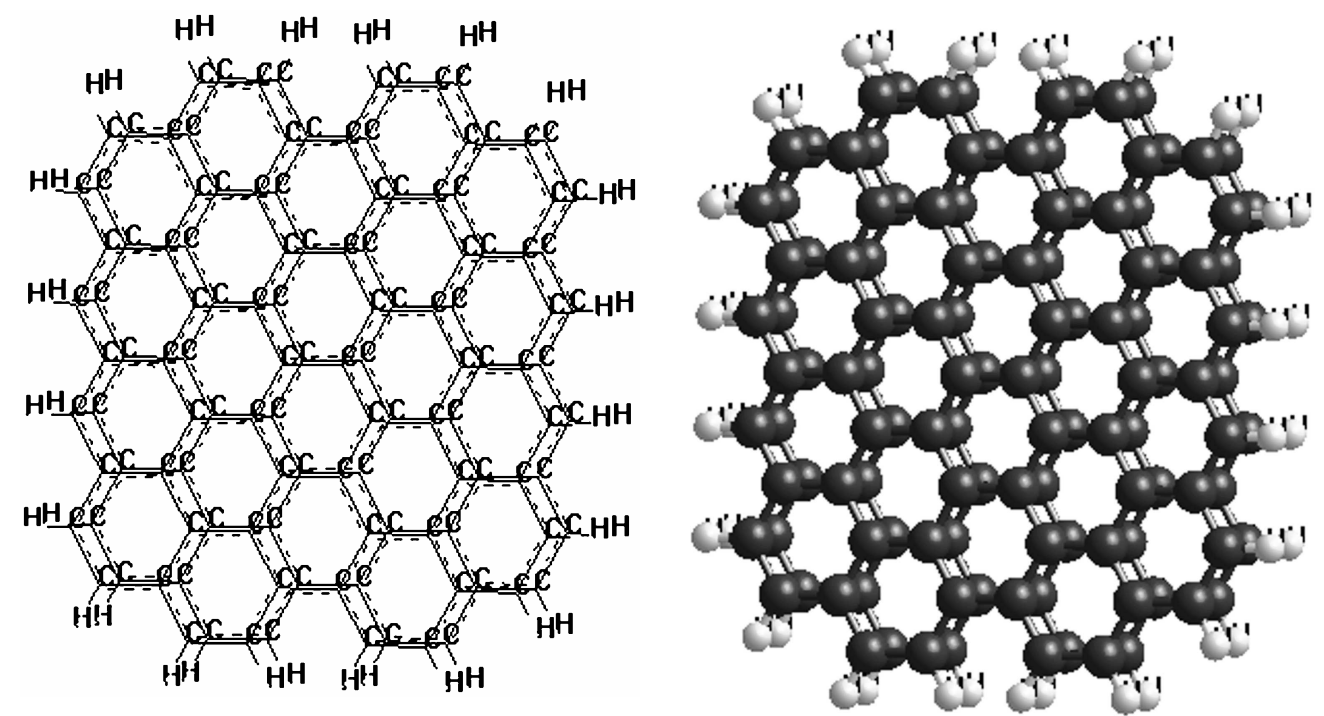

Рис. 2. Рассчитанные модели электронной структуры фрагмента двухслойного графена $\mathrm{C}_{124} \mathrm{H}_{40}$.
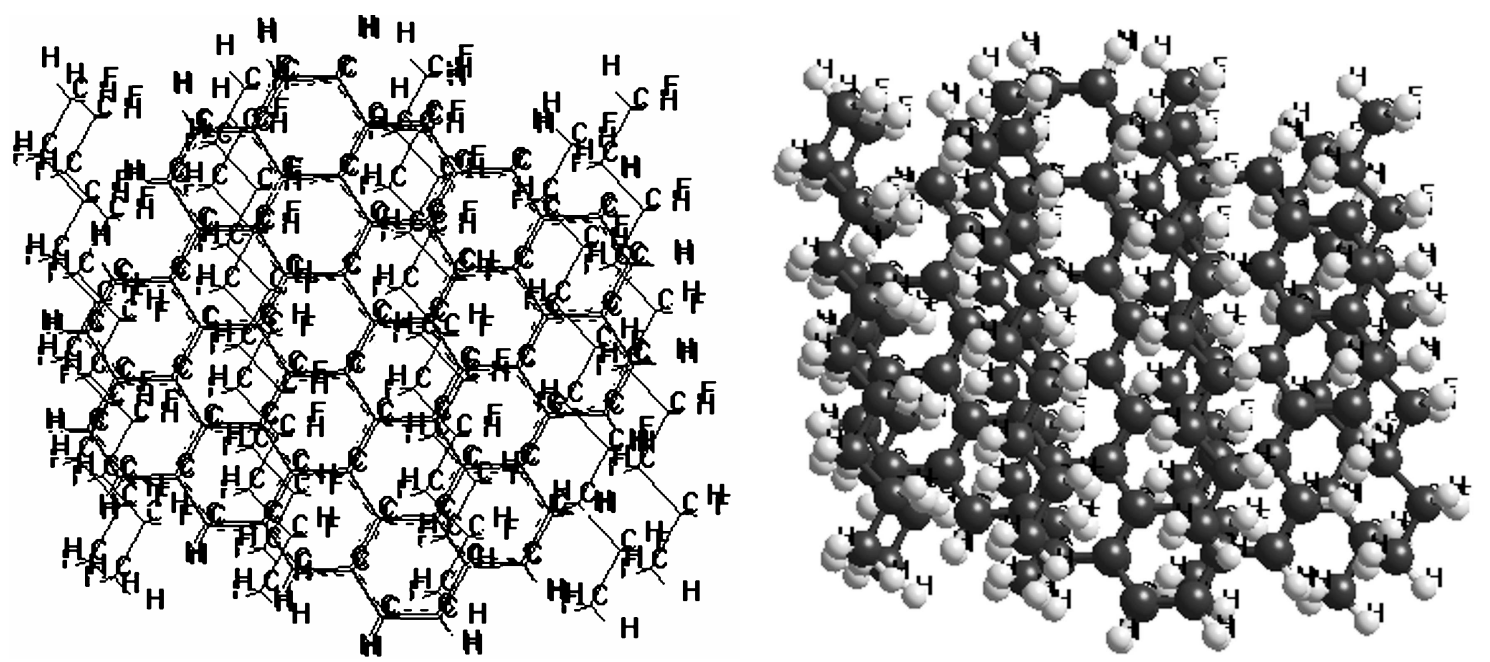

Рис. 3. Рассчитанные модели электронной структуры фрагмента двухслойного графена в полимерной матрице $\mathrm{C}_{124} \mathrm{H}_{40}+2 \mathrm{PVDF}$.

$\Delta E$ - параметр, характеризующий стабильность системы. При $\Delta E>0$ материал считается нестабильным, при $\Delta E<0$ - стабильным.

\section{3. Результаты}

На основе рассмотренных моделей композитного материала $\mathrm{C}_{124} \mathrm{H}_{40}+n\left(\mathrm{H}-\left(\mathrm{C}_{2} \mathrm{H}_{2} \mathrm{~F}_{2}\right)_{5}-\mathrm{H}\right)$, в случае, когда расстояние между слоями графена равно $0.142 \mathrm{~nm}$, вычислены значения орбитальных энергий электронных структур, потенциалы ионизации, полные энергии, жесткости, параметры стабильности, ширины запрещенных зон, длины излученных фотонов. Результаты вычислений приведены в табл. 1. Видно, что материал является электрофильным, диэлектриком и стабильным.
На рис. 2 приведены теоретические модели фрагмента двухслойного графена $\mathrm{C}_{124} \mathrm{H}_{40}$, на рис. 3 - двухслойного графена и многослойной полимерной матрицы из поливинилиденфторида $\mathrm{C}_{124} \mathrm{H}_{40}+n\left(\mathrm{H}-\left(\mathrm{C}_{2} \mathrm{H}_{2} \mathrm{~F}_{2}\right)_{5}-\mathrm{H}\right)$ $(n=\overline{1,8})$. Из табл. 1 видно, что самое высокое значение параметра жесткости $\eta=5.118 \mathrm{eV}$ наблюдается у материала $\mathrm{C}_{124} \mathrm{H}_{40}+2\left(\mathrm{H}-\left(\mathrm{C}_{2} \mathrm{H}_{2} \mathrm{~F}_{2}\right)_{5}-\mathrm{H}\right)$.

Рассчитаем величину твердости на единицу удлинения (модуль упругости Юнга) для рассматриваемого композитного материала $\mathrm{C}_{124} \mathrm{H}_{40}+2\left(\mathrm{H}-\left(\mathrm{C}_{2} \mathrm{H}_{2} \mathrm{~F}_{2}\right)_{5}-\mathrm{H}\right)$. Известно, что силу разрыва графена можно рассчитать по формуле $[1,2]$

$$
F \approx \frac{E_{b}}{r}
$$

Здесь $E_{b}-$ энергия связи рассматриваемого двухслойного графена + поливинилиденфторид, $r-$ расстояние 
Таблица 1. Результаты рассчета по РМ3-методу характеристик двухслойного графена в многослойной полимерной матрице $\mathrm{C}_{124} \mathrm{H}_{40}+n \mathrm{PVDF}(n=1,2,3,4,5,6,7,8)$

\begin{tabular}{|c|c|c|c|c|c|c|c|c|}
\hline Объект & $\begin{array}{c}\mathcal{E}_{\mathrm{HOMO} O} \\
\mathrm{amu}\end{array}$ & $\begin{array}{c}\mathcal{E}_{\mathrm{LUMO} O} \\
\mathrm{amu}\end{array}$ & $\begin{array}{c}\text { Полная } \\
\text { энергия } E, \\
\text { amu }\end{array}$ & $\begin{array}{c}\text { Параметр } \\
\text { стабильности } \Delta E, \\
\text { amu }\end{array}$ & $\begin{array}{c}\text { Потенциал } \\
\text { ионизации } \\
I_{p}(\mathrm{eV})\end{array}$ & $\begin{array}{c}\text { Ширина } \\
\text { запрещенной } \\
\text { зоны } E_{g}(\mathrm{eV})\end{array}$ & $\begin{array}{c}\text { Параметр } \\
\text { жесткости, } \\
\eta(\mathrm{eV})\end{array}$ & $\begin{array}{c}\text { Длина волны } \\
\text { излученного } \\
\text { фотона, nm }\end{array}$ \\
\hline PVDF & -12.508284 & 0.621244 & -212.193758 & -5.730 & 12.508 & 13.130 & 6.565 & 94.68 \\
\hline $\mathrm{C}_{124} \mathrm{H}_{40}$ & -9.449374 & 1.163273 & -551.9558726 & -37.850 & 9.449 & 10.613 & 5.306 & 117.14 \\
\hline $\begin{array}{l}\mathrm{C}_{124} \mathrm{H}_{40} \\
+1 \mathrm{PVDF} \\
(N=1)\end{array}$ & -9.03805 & 1.198191 & -762.3533594 & -41.786 & 9.038 & 10.236 & 5.118 & 121.44 \\
\hline $\begin{array}{l}\mathrm{C}_{124} \mathrm{H}_{40} \\
+2 \mathrm{PVDF} \\
(N=2)\end{array}$ & -9.468073 & 0.815188 & -973.9263704 & -46.894 & 9.468 & 10.283 & 5.142 & 120.89 \\
\hline $\begin{array}{l}\mathrm{C}_{124} \mathrm{H}_{40} \\
+3 \mathrm{PVDF} \\
(N=3)\end{array}$ & -8.881871 & 0.962347 & -1184.933343 & -51.438 & 8.882 & 9.844 & 4.922 & 126.28 \\
\hline $\begin{array}{l}\mathrm{C}_{124} \mathrm{H}_{40} \\
+4 \mathrm{PVDF} \\
(n=4)\end{array}$ & -9.183008 & 0.741625 & -1396.159769 & -56.202 & 9.183 & 9.925 & 4.962 & 125.26 \\
\hline $\begin{array}{l}\mathrm{C}_{124} \mathrm{H}_{40} \\
+5 \mathrm{PVDF} \\
(N=5)\end{array}$ & -9.34528 & 0.54569 & -1607.652406 & -61.230 & 9.345 & 9.891 & 4.945 & 125.68 \\
\hline $\begin{array}{l}\mathrm{C}_{124} \mathrm{H}_{40} \\
+6 \mathrm{PVDF} \\
(N=6)\end{array}$ & -9.237814 & 0.637674 & -1819.512523 & -66.627 & 9.238 & 9.875 & 4.938 & 125.86 \\
\hline $\begin{array}{l}\mathrm{C}_{124} \mathrm{H}_{40} \\
+7 \mathrm{PVDF} \\
(N=7)\end{array}$ & -7.905623 & 0.501913 & -2028.907616 & -69.557 & 7.906 & 8.408 & 4.204 & 147.86 \\
\hline $\begin{array}{l}\mathrm{C}_{124} \mathrm{H}_{40} \\
+8 \mathrm{PVDF} \\
(N=8)\end{array}$ & -7.908125 & 0.603671 & -2242.135978 & -76.324 & 7.908 & 8.512 & 4.256 & 146.05 \\
\hline
\end{tabular}

связи между атомами

$$
E_{b}=124 E_{c}+40 E_{H}-E_{2 \text { graphene+pvdf. }}
$$

$E_{c}, E_{H}$ и $E_{2 \text { graphene+pvdf }}$ - рассчитанные по РМ3-методу $[11,12]$ полные энергии углерода, водорода и двухслойного графена+поливинилиденфторид; $E_{b}-$ энергия связи двухслойного графена+поливинилиденфторид, рассчитанная по методу РМ3

$$
\begin{gathered}
E_{c}=1.74 \cdot 10^{-17} \mathrm{~J} ; \quad E_{H}=0.21 \cdot 10^{-17} \mathrm{~J} \\
E_{2 \text { graphen }+ \text { pvdf }}=2.41 \cdot 10^{-15} \mathrm{~J} \\
E_{b}=1.849 \cdot 10^{-16} \mathrm{~J}, \quad r=0.13 \cdot 10^{-9} \mathrm{~m} .
\end{gathered}
$$

Эти вычисленные значения подставляем в формулу (1), получаем величину твердости на единицу удлинения (модуль упругости Юнга) для композитного материала $\mathrm{C}_{124} \mathrm{H}_{40}+2\left(\mathrm{H}-\left(\mathrm{C}_{2} \mathrm{H}_{2} \mathrm{~F}_{2}\right)_{5}-\mathrm{H}\right)$

$$
Y=\frac{F}{S}=\frac{E_{b}}{r S} \text {. }
$$

Здесь $S$ - площадь сечения фрагмента графена, состоящего из 62 атомов углерода ${ }^{12} \mathrm{C}$. В результате получаем величину модуля Юнга рассматриваемого композитного материала

$$
Y=1.473 \mathrm{TPa}
$$

Таким же способом получаем, что если для однослойного графена энергия связи равна $E_{b}=0.79 \cdot 10^{-16} \mathrm{~J}$, тогда $Y=629.1 \mathrm{GPa}$, что если для двухслойного графена энергия связи равна $E_{b}=1.128 \cdot 10^{-16} \mathrm{~J}$, тогда $Y=897.9 \mathrm{GPa}$, то есть модуль Юнга двухслойного графена в 1.4 раза больше, чем для однослойного. А модуль Юнга $Y=1.473$ ТРа двухслойного графена с 
Таблица 2. Результаты расчета по РМЗ-методу значений модуля сдвига, модуля объемный упругости, модуля Юнга и модуля твердости графена, двухслойного графена и двухслойного графена с многослойной полимерной матрицей (PVDF)

\begin{tabular}{l|c|c|c|c}
\hline \multicolumn{1}{c|}{ Материалы } & $\begin{array}{c}\text { Модель сдвига } \\
(\mathrm{GPa})\end{array}$ & $\begin{array}{c}\text { Модуль объемной упругости } \\
(\mathrm{GPa})\end{array}$ & $\begin{array}{c}\text { Модуль Юнга } \\
(\mathrm{GPa})\end{array}$ & $\begin{array}{c}\text { Модуль твердости } \\
(\mathrm{GPa})\end{array}$ \\
\hline Графен & 312 & 870.9 & 836.1 & 130 \\
Двухслойный графен & 335 & 935.3 & 897.9 & 139.6 \\
Двухслойный графен & 549.5 & 1534 & 1473 & 228.9 \\
с композитным (PVDF) & & & &
\end{tabular}

композитным материалом в 2.3 раза больше, чем для однослойного.

А для вычисления модуля сдвига, модуля объемной упругости, модуля твердости графена, двухслойного графена и двухслойного графена с композитами используем следующие формулы [24,25]

$$
G=\frac{Y}{2(1+v)}, \quad K=\frac{Y}{3(1-2 v)}, \quad H N=G A e^{-B T} .
$$

Здесь $v=0.34$ коэффициент Пуассона [26], $Y-$ модуль Юнга, $G$ - модуль сдвига, $K$ - модуль объемный упругости, $H N$ - модуль твердости, $A=0.807$, $B=2.204 \cdot 10^{-3}$ и $T=300^{\circ} \mathrm{K}$. Результаты вычисления заданы в табл. 2.

Таким образом, рассматриваемый композитный материал на основе двухслойного графена $\mathrm{C}_{124} \mathrm{H}_{40}+2\left(\mathrm{H}-\left(\mathrm{C}_{2} \mathrm{H}_{2} \mathrm{~F}_{2}\right)_{5}-\mathrm{H}\right)$ очень твердый и легкий: $\rho \approx 1.7 \mathrm{~g} / \mathrm{cm}^{3}$. По этой причине, он может быть использован для изготовления различных твердых и, в то же время, легких материалов, в частности бронежилетов.

На рис. 1 показана схема разрыва композитного материала $\mathrm{C}_{124} \mathrm{H}_{40}+2\left(\mathrm{H}-\left(\mathrm{C}_{2} \mathrm{H}_{2} \mathrm{~F}_{2}\right)_{5}-\mathrm{H}\right)$ под действием удара пули. Из схемы видно, что под действием удара пули композитный материал удерживает пулю, однако трескается. В результате этого, образовавшиеся осколки могут повредить организм человека. Однако, слои твердого и то же время эластичного материала поливинилиденфторида захватывают и удерживают осколки графена, и не позволяют осколкам проникать в человеческое тело.

Кроме требования к бронежилету не разрушиться при действии пули, есть требование рассеять кинетическую энергию пули по большой площади, которое будет зависеть от ряда факторов, в первую очередь от теплопроводности (теплофизические свойства) графена и прочности слоя композита на изгиб. В работе [27] отмечено, что теплофизические свойства графена (в частности, теплопроводность) определяются фононами, особенности спектра которых связаны с двумерной структурой листа графена. Согласно имеющимся экспериментальным данным [28], графен имеет самую высокую среди твердых тел теплопроводность: $4840-5300 \mathrm{~W} \cdot \mathrm{m}^{-1} \cdot \mathrm{K}^{-1}$ для однослойного и $3000 \mathrm{~W} \cdot \mathrm{m}^{-1} \cdot \mathrm{K}^{-1}$ для двухслойного графена.

При ударе пули об бронежилет кинетическая энергия пули перейдет в тепловую, которая, благодаря очень высокой теплопроводности графена, с высокой эффективностью и скоростью (скорость звука в графене достигает $10 \mathrm{~km} \cdot \mathrm{sec}^{-1}$ ) рассеется по большой площади бронежилета и погаснет. Таким образом, наряду с высокой твердостью композита на изгиб, его высокая теплопроводность обеспечит стойкость бронежилета на разрыв от удара пули.

Предположим, что в результате выстрела пуля ударяется в композитный материал на основе двухслойного графена и полимерной матрицы (рис. 1). Какова должна быть толщина бронежилета $h$, чтобы пуля не пробила бы его?

Допустим, что $V$ - скорость пули при ударе об бронежилет, $m-$ масса пули, $S$ - площадь поперечного сечения соприкосновения пули с бронежилетом. После удара, можно предположить, что пуля в бронежилете двигается с равнозамедленным ускорением и, пройдя расстояние $h$, останавливается. В это время, пуля оказывает давление $p$ на бронежилет. Условие непробиваемости бронежилета можно записать так:

$$
p<H N=228.9 \mathrm{GPa} \text {. }
$$

Используя известные формулы из кинематики и динамики легко получаем условие

$$
p=\frac{m V^{2}}{2 h S}<H N=228.9 \mathrm{GPa} .
$$

Рассмотрим два случая: выстрел из автомата Калашникова АКМ и пистолета Макарова ПМ.

1. Для АКМ имеем: $V=600 \mathrm{~m} / \mathrm{sec}, m=0.009 \mathrm{~kg}$, диаметр сечения острия пули $D=1 \mathrm{~mm}$. Тогда, используя формулу (10), определяем минимальную „непробиваемую“ для пули АКМ толщину бронежилета

$$
\min (h)=0.0090111318 \mathrm{~m} \text {. }
$$

Для расчета массы бронежилета воспользуемся формулой

$$
M=a b h \rho .
$$

Здесь $a, b, h$ - габариты бронежилета, $\rho$ - плотность графена.

Если возьмем: $a=0.3 \mathrm{~m}, b=0.4 \mathrm{~m}, h=0.0090111318 \mathrm{~m}$, $\rho \approx 1.7 \mathrm{~g} / \mathrm{cm}^{3}$, тогда согласно формуле (11) получим $M=1.8382708814 \mathrm{~kg}$. 
2. Для ПМ имеем: $V=300 \mathrm{~m} / \mathrm{sec} m=0.009 \mathrm{~kg}$, диаметр сечения острия пули $D=9 \mathrm{~mm}$. Тогда, используя формулу (10), определяем минимальную „непробиваемую“ для пули ПМ толщину бронежилета: $\min (h)=0.0000278121 \mathrm{~m}$, а массу бронежилета согласно формуле (11) получим $M=0.0056736756 \mathrm{~kg}$.

Таким образом, при использовании сверхпрочного композитного материала $\mathrm{C}_{124} \mathrm{H}_{40}+2\left(\mathrm{H}-\left(\mathrm{C}_{2} \mathrm{H}_{2} \mathrm{~F}_{2}\right)_{5}-\mathrm{H}\right)$, бронежилет получается очень тонким и легким.

\section{4. Заключение}

Таким образом, в статье применяя РМ3-метод исследована электронная структура ударопрочного материала $\mathrm{C}_{124} \mathrm{H}_{40}+n\left(\mathrm{H}-\left(\mathrm{C}_{2} \mathrm{H}_{2} \mathrm{~F}_{2}\right)_{5}-\mathrm{H}\right)(n=\overline{1,8})$ на основе двухслойного графена в многослойной полимерной матрице и построены теоретические модели. На основе моделей при допущении, что $\mathrm{C}-\mathrm{C}$-расстояние равно $0.13 \mathrm{~nm}$, а расстояние между слоями графена равно $0.142 \mathrm{~nm}$, вычислены значения орбитальных энергий электронных структур, потенциалы ионизации, полные энергии, жесткости, параметры стабильности, ширины запрещенных зон, длины излученных фотонов, прочность для этих материалов. Расчеты показали, что рассматриваемый материал на основе двухслойного графена является электрофильным, диэлектриком, стабильным и в 1.761 раза прочнее однослойного графена. Для самой прочной модели композитного материала на основе двухслойного графена $\mathrm{C}_{124} \mathrm{H}_{40}+2\left(\mathrm{H}-\left(\mathrm{C}_{2} \mathrm{H}_{2} \mathrm{~F}_{2}\right)_{5}-\mathrm{H}\right)$ (модель $N=2$ ) получили значение твердости (прочности) $228.9 \mathrm{GPa}$.

Проведенные расчеты показали, что данный композитный материал можно использовать в военной области для изготовления сверхпрочных и легких бронежилетов, различных сверх прочных покрытий. Проведены оценки минимальных толщин и масс бронежилетов, изготовленных из рассматриваемых композитных материалов, способных выдержать удары пуль, выпущенных из автомата АКМ и пистолета ПМ.

\section{Список литературы}

[1] M.I. Katsnelson. Graphene: Carbon in Two Dimensions. Cambridge University Press. N.Y. (2012). 366 p.

[2] A.H. Castro Neto, F. Guinea, N.M.R. Peres, K.S. Novoselov, A.K. Geim. Rev. Mod. Phys. 81, 109 (2009).

[3] A.K. Geim, K.S. Novoselov. Nature Mater. 6, 182 (2007).

[4] С.Ю. Давыдов. ФТТ 58, 392 (2016).

[5] K.S. Novoselov, E. McCann, S.V. Morozov, V.I. Fal'ko, M.I. Katsnelson, U. Zeitler, D. Jiang, F. Schedin, A.K. Geim. Nature Phys. 2, 177 (2006).

[6] J.S. Bunch, M.Z. Arend, S.S. Verbridge, I.W. Frank, D.M. Tanenbaum, J.M. Parpia, H.G. Craighead, P.L. McEuen. Science 315, 490 (2007).

[7] D.A. Abanin, S.V. Morozov, L.A. Ponomarenko, R.V. Gorbachev, A.S. Mayorov, M.I. Katsnelson, K. Watanabe, T. Taniguchi, K.S. Novoselov, L.S. Levitov, A.K. Geim. Science 332, 328 (2011).
[8] https://www.innoros.ru/news/14/12/bronezhilety-iz-grafena

[9] Ali R. Ranjbartoreh, Bei Wang, Xiaoping Shen, Guoxiu Wang. J. Appl. Phys. 109, 014306 (2011).

[10] V.G. Zavodinsky E.A. Mikhailenko. Phys. Low-Dimensional Structures 5/6, 35 (2004).

[11] V.G. Zavodinsky, I.A. Kuyanov, E.N. Chukurov. Surf. Rev. Lett. 6, 1, 127 (1999).

[12] В.Г. Заводинский, А.Н. Чибисов, А.А. Гниденко, М.А. Алейникова. Механика композиционных материалов и конструкций 11, 3, 337 (2005).

[13] Е.А. Беленков, В.А. Грешняков. ФТТ 57, 192 (2015).

[14] M.A. Ramazanov, A.G. Gasanov, F.G. Pashaev, M.R. Vahabova. Fizika XXIII, 9 Section: Az (2017).

[15] A.G. Gasanov, A.A. Bayramov, E.G. Hashimov. Fizika XXIII, 34 Section, (2017).

[16] M.A. Ramazanov, A.G. Gasanov, F.G. Pashaev. Fizika XXIII, 3 Section: Az (2017).

[17] A.M. Maharramov, M.A. Ramazanov, A.G. Gasanov, F.G. Pashaev. Phys. Sci. Int. J. 10, 3, 1 (2016).

[18] M.A. Ramazanov, F.G. Pashaev, A.G. Gasanov, A.M. Maharramov, A.T. Mahmood. Chalcogenide Lett. V11, 7, 359 (2014).

[19] F.G. Pashaev, A.G. Gasanov, A.T. Mahmood. J. Nano. Adv. Mater. 2, 1, 35 (2014).

[20] J.J.P. Stewart, I. Method. J. Comp. Chem. 10, 2, 209 (1989).

[21] A.G. Gasanov. Изв. БГУ. Сер. физ.-мат. 2, 171 (2011).

[11] A.G. Gasanov, F.G. Pashaev. Romanian J. Informa. Sci. Technol. 19, 4, 331 (2016).

[23] В.И. Минкин, Б.Я. Симкин, Р.М. Минаев. Теория структуры молекулы. Phoenix, Ростов н/Д (1997). 558 с.

[24] Т.П. Черняева, В.М. Грицина, Е.А. Михайлов, А.В. Остапов. Корреляция между упругостью и другими свойствами циркония. Научно-техн. комп. „Ядерный топливный цикл“. ННЦ Харьковский физ.-тех. институт, Харьков, Украина (2008).

[25] Л.А. Опенов 1, А.И. Подливаев. ФТТ 59, 6, 1240 (2017).

[26] J.-W. Jiang, H.S. Park. Nano Lett. 16, 2657 (2016).

[27] А.В. Елецкий, И.М. Искандарова, А.А. Книжник, Д.Н. Красиков. УФН 181, 233 (2011).

[28] S. Ghosh, I. Calizo, D. Teweldebrhan, E.P. Pokatilov, D.L. Niko, A.A. Balandin, W. Bao, F. Miao, C.N. Lau. Appl. Phys. Lett. 92, 151911 (2008).

Редактор Т.Н. Василевская 